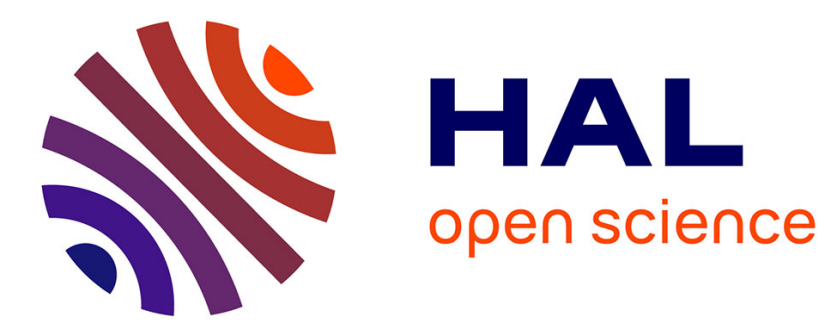

\title{
Les vergers à graines de l'Etat dans le Lot
}

Inra Centre de Recherches Forestières d'Orléans, Ctgref Centre Technique Du

Génie Rural Des Eaux Et Forêts

\section{To cite this version:}

Inra Centre de Recherches Forestières d'Orléans, Ctgref Centre Technique Du Génie Rural Des Eaux Et Forêts. Les vergers à graines de l'Etat dans le Lot. Revue forestière française, 1975, 27 (5), pp.350-356. 10.4267/2042/20955 . hal-03395529

\section{HAL Id: hal-03395529 \\ https://hal.science/hal-03395529}

Submitted on 22 Oct 2021

HAL is a multi-disciplinary open access archive for the deposit and dissemination of scientific research documents, whether they are published or not. The documents may come from teaching and research institutions in France or abroad, or from public or private research centers.
L'archive ouverte pluridisciplinaire HAL, est destinée au dépôt et à la diffusion de documents scientifiques de niveau recherche, publiés ou non, émanant des établissements d'enseignement et de recherche français ou étrangers, des laboratoires publics ou privés. 


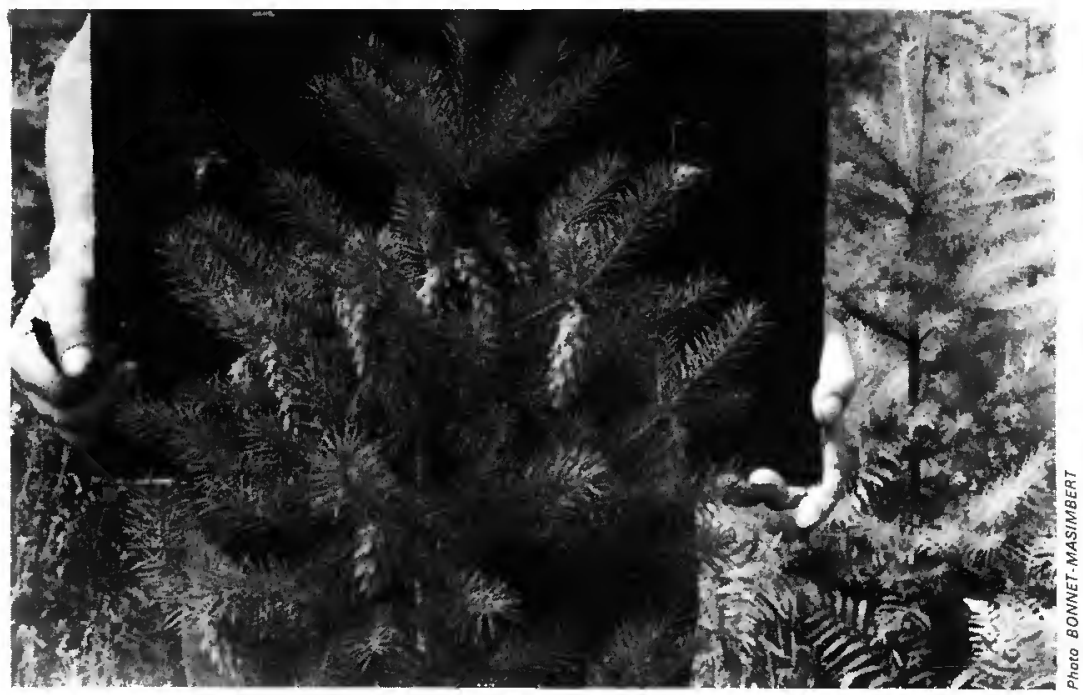

Douglas fructifere de 4 ens dans le verger à graines de familles de Cendrieux (Dordogne)

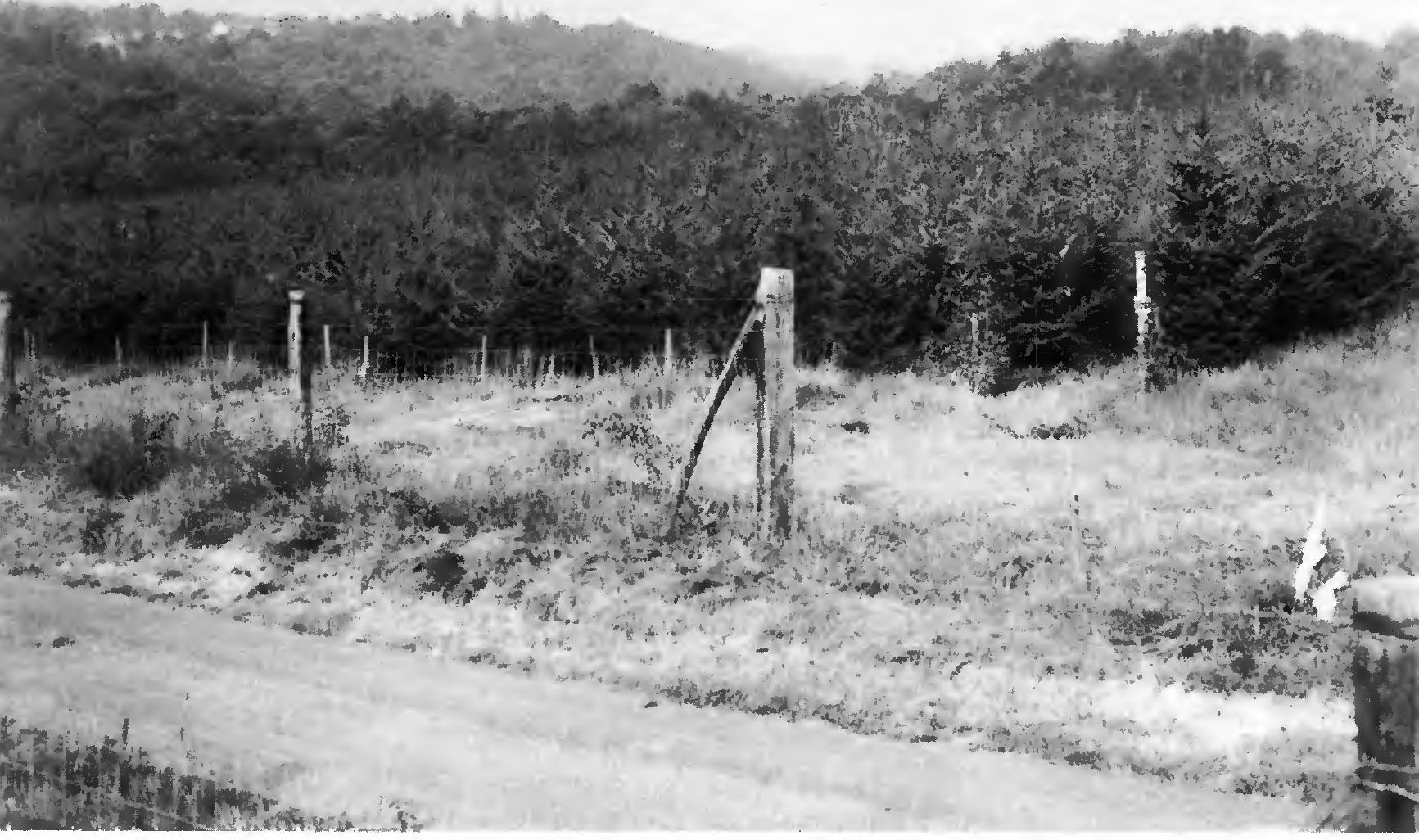

Vue d'ensemble d’un verger à graines de familles da 4 ans de Douglas-Cendrieux (Dordogne)

PhOto LANARES 


\title{
LES VERGERS A GRAINES DE L'ÉTAT DANS LE LOT *
}

\author{
C. T. G. R. E. F. - I. N. R. A.
}

Class. Oxford $232.311 .3(44 \times 13)$

Alors que les semences agricoles sont produites dans des champs spécialisés, les graines forestières sont encore récoltées sur des peuplements dont le but essentiel est la production de bois.

Grâce aux travaux menés depuis quelques décennies, tant en France qu'à l'étranger, par les instituts de génétique forestière, il est maintenant possible d'entamer véritablement la production de variétés forestières synthétiques offrant, par rapport aux variétés naturelles, une supériorité génétique substantielle.

Tel est l'objectif principal du programme de vergers à graines de l'État dans le Lot.

\section{RAPPEL SUR LES VERGERS À GRAINES FORESTIERS}

\section{- Généralités}

Des trois méthodes classiques de l'amélioration génétique des espèces : la sélection, l'hybridation et la mutation, seules les deux premières ont jusqu'à ce jour débouché, en matière forestière, sur des résultats concrets. Encore faut-il préciser, qu'excepté le cas important des mélèzes, les recherches sur les hybrides ont surtout été faites par les généticiens américains et ne concernent pratiquement pas les espèces de reboisement utilisées en France.

La sélection constitue donc l'outil privilégié d'amélioration génétique des arbres forestiers. Elle s'effectue en deux étapes :

- sélection dans l'aire naturelle ou artificielle de l'espèce des races géographiques les mieux adaptées aux conditions de reboisement étudiées, ou sélection de provenances:

- sélection dans les meilleures provenances des individus présentant, au plus haut point, les qualités recherchées ou sélection individuelle.

(*) Extraits mis à jour du document n $n^{\circ} 10$ remis à l'occasion du voyage d'étude organisé en France du 21 au 26 octobre 1974 dans le cadre de la dixième session et du colloque commun du Comité mixte et de l'Organisation internationale des instituts de recherches forestières. 
Pour essentielle que soit, dans le déroulement d'un programme d'amélioration, l'étape de la sélection de provenances, ce n'est que l'étape de la sélection individuelle qui va permettre de déboucher sur la variété artificielle par la création de vergers à graines.

Selon que l'on envisage ou non de poursuivre, dans le verger, les sélections individuelles déjà opérées sur le matériel de départ (matériel de base) deux types de vergers vont pouvoir être créés: les vergers de clones et les vergers de familles.

\section{- Caractéristiques des divers types de vergers}

\section{Vergers à graines de clones}

Le verger à graines de clones est une plantation de «copies végétatives 》 d'arbres sélectionnés (copies obtenues en général par greffage ou bouturage).

Dans un tel verger, le gain génétique espéré ne dépend que de la valeur et de l'intensité de la sélection pratiquée sur le matériel de départ; il ne peut, en principe, être amélioré durant cette phase qui est seulement une phase de production.

Ainsi dans un verger de clones d'arbres plus, le gain génétique est-il discutable chaque fois que les caractères que l'on cherche à améliorer n'offrent que peu de prise à la sélection individuelle en forêt, ce qui est malheureusement le cas de caractères intéressants tels que la vigueur.

Ce problème ne se pose évidemment pas dans le cas de vergers d'arbres d'élite (1), mais compte tenu des délais et des moyens nécessaires pour repérer les arbres d'élite, ce type de verger n'est pas encore susceptible de déboucher, en pratique, sur des réalisations importantes.

Les éclaircies étant nulles ou de faible importance dans le verger de clones, son installation, à une densité voisine de la densité définitive (300 à 400 plants à l'hectare), et sa gestion sont, en général, simples.

Les tailles fructifères ou de formation peuvent y être entreprises précocement ce qui, joint à l'effet de greffe, peut conduire à une accélération de la mise à fruits et facilitera, en tout cas, l'exécution des récoltes.

Le verger de clones peut être implanté dans des régions écologiquement très différentes de celles pour lesquelles sa production est adaptée, en particulier dans des zones favorables à la fréquence et à l'abondance des fructifications.

En contrepartie, il s'adapte mal au cas des espèces qui posent des problèmes de multiplication végétative (difficulté de greffage ou, ce qui est pire, incompatibilité à manifestation plus ou moins tardive entre le greffon et le porte-greffe, comme c'est le cas du Douglas).

\section{Vergers à graines de familles}

Le verger à graines de familles est une plantation comparative de descendance (2) à structure monoarbre dans laquelle des éclaircies génétiques sont périodiquement effectuées et où, en définitive, ne sont conservés que les meilleurs sujets des meilleures familles.

Dans un tel verger, le gain génétique espéré dépend non seulement de la valeur du matériel de départ, mais également de la qualité et de l'intensité de la sélection qui sera réalisée dans le verger lui-même, si bien que ce dernier a une double fonction : production de masse et sélection.

(1) Un arbre d'élite est un arbre plus qui a fait la preuve de sa supériorité génétique.

(2) Une descendance est un ensemble d'individus ayant en commun au moins l'un des deux parents. 
Ce double rôle introduit, dans l'installation et la gestion du verger de familles, des contraintes particulières :

- délimitation des zones d'égale fertilité du terrain (cartographie de fertilité) pour asseoir correctement les répétitions et permettre des comparaisons valables entre les familles et à l'intérieur des familles ;

- installation à une densité suffisante (2 500 à 5000 plants à I'hectare) pour autoriser une bonne intensité d'éclaircies:

- repérage individuel des plants;

- exécution tout au long de la période de sélection, de nombreuses mensurations ou cotations ;

- exploitation et stockage de l'information.

Pour écarter le risque d'une sélection faussée, le verger de famille doit en outre être implanté dans un milieu écologiquement voisin de celui auquel sa production est destinée.

\section{Vergers à graines hybrides}

Ce type de verger où l'espoir d'amélioration génétique repose essentiellement sur une possible manifestation de la vigueur hybride (hétérosis) ne concerne, dans le programme de vergers à graines du Lot, qưune seule essence : le mélèze.

La graine hybride sera, en l'occurrence, obtenue par fécondation d'un clone autostérile de Mélèze d’Europe sélectionné dans une provenance polonaise par le pollen de plusieurs clones bien individualisés de Mélèze du Japon appartenant d'ailleurs à la même famille.

La graine « utile » (Larix eurolepis) ne sera donc récoltée que sur le clone de Mélèze d'Europe. Déjà testée, elle a comme caractéristique de donner des peuplements homogènes et vigoureux.

\section{LE PROGRAMME DES VERGERS DU LOT}

Pour la réalisation de ce programme, financé principalement par le Fonds forestier national, une convention a été passée entre l'État (Service des forêts) et l'Office national des forêts dans laquelle l'Office national des forêts (Direction régionale Midi-Pyrénées, Centre de Toulouse) est chargé de l'installation, de l'entretien et de la gestion des vergers à graines. La direction technique des travaux est assurée par le Centre technique du génie rural, des eaux et des forêts (C.T.G.R.E.F.-Division "Graines et plants forestiers »), qui bénéficie, pour ce faire, de la collaboration de la Station d'amélioration des arbres forestiers (Orléans) et du Laboratoire d'amélioration des conifères (Bordeaux), tant pour ce qui est de la définition ou de la mise au point de la méthodologie d'installation et de sélection dans les vergers, que pour ce qui est de la fourniture des matériels de base.

\section{- Liste des principaux vergers projetés}

Le programme porte sur la création d'environ 240 ha de vergers ainsi répartis : 


\begin{tabular}{|c|c|c|c|c|}
\hline Essence & Matériel de base & $\begin{array}{l}\text { Débouché normal } \\
\text { de la graine du verger }\end{array}$ & $\begin{array}{c}\text { Type }\left(^{*}\right) \\
\text { de } \\
\text { verger }\end{array}$ & $\begin{array}{c}\text { Superficie } \\
\text { (approximative) } \\
\text { ha }\end{array}$ \\
\hline $\begin{array}{l}\text { Épicéa } \\
\text { commun }\end{array}$ & 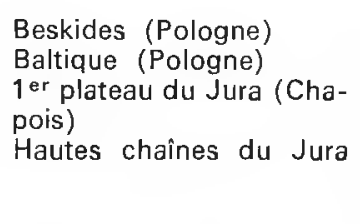 & $\begin{array}{l}\text { Basse et moyenne altitude } \\
\text { Basse altitude } \\
\text { Basse et moyenne altitude } \\
\text { Haute altitude }\end{array}$ & $\begin{array}{l}\mathrm{F} \\
\mathrm{F} \\
\mathrm{F} \\
\mathrm{C}\end{array}$ & $\begin{array}{l}10 \\
15 \\
25 \\
10 \\
60\end{array}$ \\
\hline Douglas & $\begin{array}{l}\text { Peuplements français } \\
\text { Piedmond des Cascades } \\
\text { Washington }\end{array}$ & $\begin{array}{l}\text { Basse et moyenne altitude } \\
\text { Basse et moyenne altitude }\end{array}$ & $\begin{array}{l}\mathrm{C} \\
\mathrm{F}\end{array}$ & $\begin{array}{r}50 \\
10 \\
60\end{array}$ \\
\hline $\begin{array}{l}\text { Pin laricio de } \\
\text { Corse }\end{array}$ & $\begin{array}{l}\text { Corse } \\
\text { Peuplements français }\end{array}$ & & $\begin{array}{l}F \\
F\end{array}$ & $\begin{array}{l}10 \\
60 \\
70\end{array}$ \\
\hline Pin maritime & Landes de Gascogne & $\begin{array}{l}\text { Reboisement hors zone } \\
\text { landaise }\end{array}$ & $\mathrm{F}$ & 40 \\
\hline \multirow[t]{2}{*}{ Mélèze hybride } & \multirow[t]{2}{*}{$\begin{array}{cc}\text { Larix decidua (Pologne) } & \times \\
& \\
\text { Larix leptolepis (Japon) }\end{array}$} & \multirow[t]{2}{*}{ Basse altitude } & \multirow[t]{2}{*}{$\mathrm{H}$} & 10 \\
\hline & & & & Total 240 \\
\hline
\end{tabular}

Ce programme appelle les remarques et justifications ci-après :

- la liste des espèces améliorées a été choisie en fonction de l'importance présente ou prévisible que ces essences ont ou auront dans les reboisements français ainsi que de l'état actuel d'avancement des travaux de sélection (ce dernier point $n$ 'étant évidemment pas sans liens avec le précédent);

- les vergers de familles ont, d'une façon générale, été préférés aux vergers de clones tant en raison des problèmes de multiplication végétative qui se posent chez les espèces autres que les pins et les mélèzes, que par suite du niveau d'amélioration limité présenté par les matériels de départ actuellement disponibles, notamment en ce qui concerne des critères essentiels tels que la rapidité de croissance; toutefois, pour le Douglas, du fait de la rareté des fructifications sur les arbres sélectionnés dans les peuplements français, de la disparition par exploitation chaque année d'environ $10 \%$ de l'effectif sélectionné, et de la durée limitée de conservation des graines, la constitution du stock de semences nécessaires à la création d'un verger de familles est apparue problématique si bien qu'il a été décidé de substituer au verger de semis un verger de clones, les boutures étant préférées aux greffes en raison du phénomène de rejet tardif de la greffe souvent constaté chez le Douglas;

- les conditions climatiques régnant dans les régions choisies pour l'implantation des vergers (Ségala du Lot pour l'épicéa et le Douglas, Bourianne pour le pin maritime et le pin laricio de Corse) sont suffisamment proches de celles existant dans les principales régions d'utilisation de ces diverses espèces pour que le risque de sélection «faussée " évoqué précédemment puisse, a priori, être considéré comme négligeable; des tests de descendances, installés par I'I.N.R.A. et par d'autres stations, permettront d'ailleurs de déceler d'éventuelles interactions et ainsi de définir les zones d'utilisation possible de la graine des vergers; 


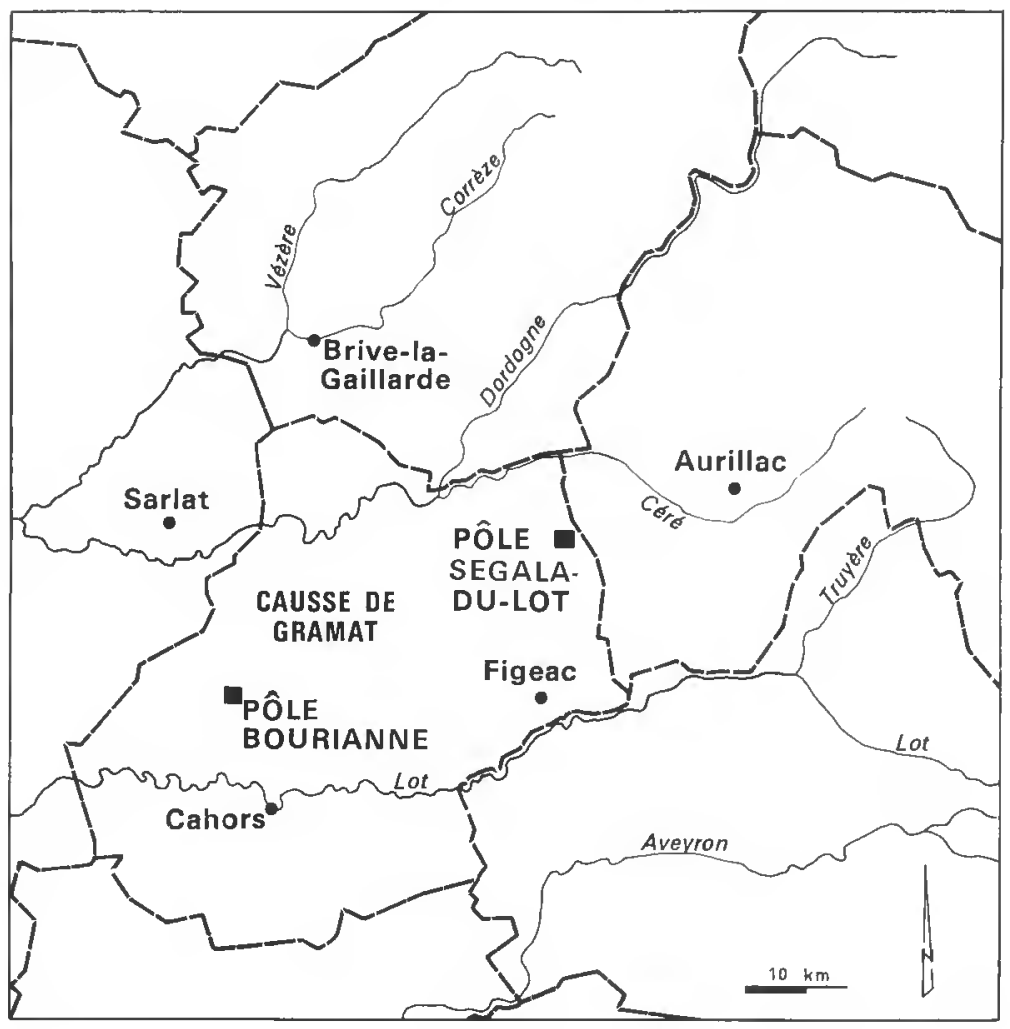

- le raisonnement ci-dessus ne peut néanmoins valablement s'appliquer lorsqu'il s'agit de régions d'utilisation particulières, telles que la haute ou très haute altitude en matière d'épicéa : comme la production de graines d'épicéa adaptée à la haute altitude s'avérait souhaitable, il est apparu nécessaire, dans ce cas particulier, de s'en tenir à la technique du verger de clones.

\section{Production escomptée}

Les délais prévus pour la mise à fruit varient selon les espèces :

$$
\begin{array}{r}
15 \text { à } 20 \text { ans pour l'épicéa, } \\
10 \text { à } 15 \text { ans pour le Douglas, } \\
8 \text { à } 10 \text { ans pour le pin maritime, } \\
10 \text { à } 15 \text { ans pour le pin laricio de Corse, } \\
5 \text { à } 10 \text { ans pour le mélèze hybride. }
\end{array}
$$

Les récoltes moyennes annuelles espérées en période de pleine production sont de

$$
\begin{aligned}
& 600 \mathrm{~kg} \text { de graines d'épicéa . . . . . . . . (10 kg/ha/an) } \\
& 600 \mathrm{~kg} \text { de graines de Douglas . . . . . . . . (10 kg/ha/an) } \\
& 1600 \mathrm{~kg} \text { de graines de pin maritime . . . . . . (40 kg/ha/an) } \\
& 1400 \mathrm{~kg} \text { de graines de pin laricio de Corse . . (20 kg/ha/an) } \\
& 50 \mathrm{~kg} \text { de graines de mélèze hybride .. . ( } 5 \mathrm{~kg} / \mathrm{ha} / \mathrm{an})
\end{aligned}
$$


Cette production ne suffira pas à satisfaire l'intégralité de nos besoins mais c'est volontairement que le premier programme a été limité, laissant ainsi la place pour de nouvelles générations de vergers qui intégreront les résultats des recherches en cours ou à venir.

\section{- Production annexe}

Une partie non négligeable des terrains acquis ne répondent pas aux conditions nécessaires pour l'implantation de vergers de familles (homogénéité de fertilité, pentes), les zones ainsi disponibles seront utilisées pour l'installation de peuplements porte-graines de bonnes provenances d'espèces autres que celles du verger et ne s'hybridant pas avec elles (Abies grandis, Picea sitchensis, etc.).
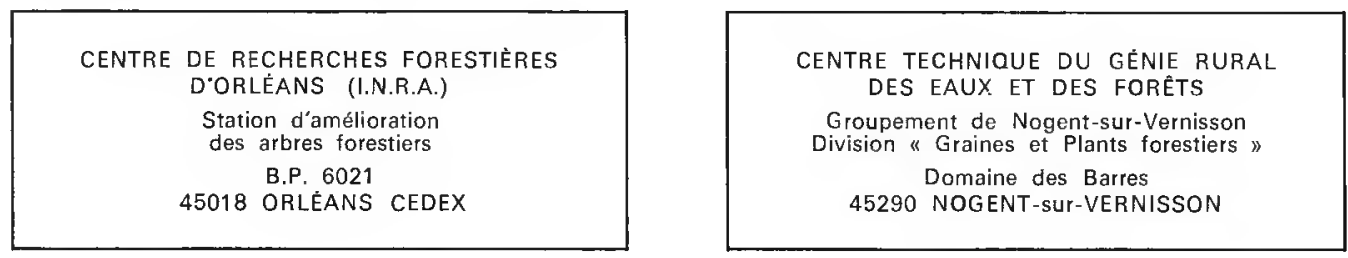

\section{$\begin{array}{llllllllllll}\text { LE S } & \text { P U B L I C A T I O N S } & \text { D U } & \text { C. } & \text { T. } & \text { G. } & \text { R. E. F. }\end{array}$}

- Le Groupement Technique Forestier du Centre technique du gẻnie rural, des eaux et des forêts (Division "Techniques de reboisement ») vient de publier une note technique intitulée :

\section{Reboisement - Matériels mécaniques \\ Note technique, $\mathrm{n}^{\circ} 29,2^{\text {e }}$ édition, juillet 1975}

Cette note fait l'inventaire des matériels mécaniques utilisables pour le reboisement et fournit, pour chaque type d'appareil, les indications nécessaires concernant leurs caractéristiques techniques, leur utilisation et leur rendement. Un chapitre spécial est consacré à une présentation générale des tracteurs. Des renseignements pratiques concernant le prix des matériels ainsi que les fabricants et importateurs figurent en annexe.

II s'agit d'une remise à jour de la note de même objet publiẻe en juin 1967, travail rendu nècessaire du fait de l'évolution des matériels et des techniques. 